\title{
Features and Outcomes of Spontaneous Bacterial Peritonitis in Egypt, Single Center Experience
}

\author{
Khaled A. Gameel'1, Maha M. Elsabaawy¹, Mahmoud A. El Tahawy¹, Azza M. Abd-Elaziz², \\ Imam A. Waked ${ }^{1}$ \\ ${ }^{1}$ Department of Hepatology, National Liver Institute, Menofia University, Shebin El Koum, Egypt \\ ${ }^{2}$ Department of Microbiology and Immunology, National Liver Institute, Menofia University, Shebin El Koum, Egypt \\ Email: tahawy1000@hotmail.com
}

How to cite this paper: Gameel, K.A., Elsabaawy, M.M., El Tahawy, M.A., AbdElaziz, A.M. and Waked, I.A. (2016) Features and Outcomes of Spontaneous Bacterial Peritonitis in Egypt, Single Center Experience. Open Journal of Gastroenterology, 6, 406-417.

http://dx.doi.org/10.4236/ojgas.2016.612043

Received: November 2, 2016

Accepted: December 18, 2016

Published: December 21, 2016

Copyright $\odot 2016$ by authors and Scientific Research Publishing Inc. This work is licensed under the Creative Commons Attribution International License (CC BY 4.0).

http://creativecommons.org/licenses/by/4.0/

\begin{abstract}
Spontaneous bacterial peritonitis (SBP) is a serious complication of liver cirrhosis, with a recurrence rate up to $70 \%$ at $1 \mathrm{yr}$. Diagnosis of SBP is based on a positive ascitic fluid (AF) culture and/or a polymorph nuclear leukocyte count of $>250$ cells $/ \mu \mathrm{L}$. Third generation cephalosporins had been viewed as the drug of choice. Aim of the work: to study features, and outcomes of spontaneous bacterial peritonitis in National Liver Institute (Menofia University), a single center study. Methods: This work was conducted on 60 patients had SBP. All patient had AF analysis, bedside AF culture, Blood culture (BC), Serum albumin, Ascitic fluid gradient (SAAG). Results: The more severe the liver disease, as assessed by Child-Turcotte-Pugh (CTP score), the higher the possibility to have positivity of AF culture. Patient who had positive $\mathrm{BC}$ had significant rising of Creatinine. The pattern of infection in AF culture is close to that of $\mathrm{BC}$, but Streptococci take the upper hand in $\mathrm{BC}$ and Staphylococcus in AF culture. Gram-positive organisms are more common in both $\mathrm{AF}$ and $\mathrm{BC}$ than gram negative. Mortality rate was higher in culture positive SBP patients $46.2 \%$, and BC positive patients, $62.5 \%$. In all positive cultures patients, Staph Species represents $44.8 \%$ with mortality rate of $42 \%$ of all mortalities. Most of them were resistant to cephalosporin $62.5 \%$. Strep species represents $31 \%$ with mortality rate of $20 \%$. E. coli represents $24.2 \%$ with mortality rate of $20 \%$. Conclusion: The microbial pattern of organisms responsible for SBP has changed with predominance of gram-positive organisms. Pattern of microbial infection is similar in both blood and ascetic fluid culture, so BC results can be relied upon to guide therapy, in negative AF culture.
\end{abstract}




\section{Keywords}

SBP, Ascites, Liver Failure, Bacterial Peritonitis

\section{Introduction}

Spontaneous bacterial peritonitis (SBP) is defined as infection of the intra peritoneal fluid without evidence of perforation or an intra abdominal sepsis or potential septic foci [1]. Spontaneous bacterial peritonitis is considered as treatable complication of cirrhosis that has been for long time locked at as lethal condition with mortality rate of $90 \%$ [2]. Child-Turcotte-Pugh class $\mathrm{C}$ patients having SBP constitute 70\%, and usually have poor prognosis [3] [4]. Spontaneous bacterial peritonitis (SBP) reported to have a very high recurrence rate in terminal liver disease population, reaches around $70 \%$ at $1 \mathrm{yr}$. [1]. Regardless of the recent advances in diagnosis, prevention, and treatment of SBP, it has been reported to have a high mortality ranging from $40 \%$ to $70 \%$ [3]. SBP pathogens are related to the process of bacterial translocation (BT), where viable or non-viable bacteria and bacterial products, cross the intestinal lumen to the regional and extraabdominal lymph nodes [5]. BT to mesenteric lymph nodes (MLN) has widely believed to be responsible for flare of the already present hyper dynamic circulatory state in the cirrhotic patient as well as in the development of spontaneous bacterial peritonitis [6].

There are certain additional factors in favor of BT in cirrhosis; the intestinal bacterial overgrowth, the alterations of the intestinal mucosal barrier and the deficiencies of the local immune response [7].

The diagnosis of SBP usually fulfilled with either positive ascitic fluid culture, or a polymorph nuclear leukocyte (PMNL) count of $>250$ cells $/ \mu \mathrm{L}$, or both, in addition to symptoms/signs of spontaneous bacterial peritonitis [8]. Risk Factors of SBP include decompensated cirrhosis and history of SBP [9]. Patients with low complement level have a greatest risk for SBP and those who have low protein levels in the ascitic fluid $(<1 \mathrm{~g} / \mathrm{dL})$ have a 10 -fold higher risk of developing SBP [3]. The association between gastrointestinal bleeding and bacterial infection, that is believed to be related to invasive diagnostic or therapeutic procedures, increase bacterial translocation, depression of the reticuloendothelial system functions caused by hypovolemia and shrinkage of complement levels, that may predispose bleeding patients with cirrhosis to bacteremia [10].

Empirical anti biotic therapy for spontaneous bacterial peritonitis (SBP), consists of third generation cephalosporins [11].

\section{Aim of the Work:}

The aim of this work is to study features, and outcomes of spontaneous bacterial peritonitis in National Liver Institute (Menofia University), a single center study. 


\section{Material and Methods}

This work was carried out in February and July 2010. The study was conducted on 60 patients with liver cirrhosis complicated by ascites, diagnosed as SBP with a polymorph nuclear leukocyte (PMNL) count of $>250$ cells $/ \mu \mathrm{L}$ in the ascetic fluid sample (AF), or positive AF culture. Written consent and National Liver Institute IRB (ethical committee) approval were obtained. Review of medical records showed all patients had HCV related cirrhosis and associated with past history of schistosomiasis. Exclusion criteria: We excluded from this study, Patients with secondary bacterial peritonitis and non-cirrhotic causes of ascites such as tuberculosis or malignancy, patients received systemic antibiotics for any other infection (s) or exposed to paracentesis or endoscopy within 7 days.

All subjects enrolled in the study were subjected to, Full medical history and medical examination, Liver functions tests (total proteins, albumin, AST, ALT, total and direct serum bilirubin, alkaline phosphatase and prothrombin time), Kidney functions tests (serum creatinine, blood urea nitrogen (BUN), Serum electrolytes (serum sodium and potassium), Complete blood count, ascetic fluid analysis (Biochemical, Cytological, gram stain, total and differential cell counts, and modified culture method, or bedside ascetic fluid culture on blood culture medium), Blood culture, Serum albumin, ascitic fluid gradient (SAAG), and Abdominal Ultrasonography.

Following collection of samples for ascetic fluids and blood culture, the patients was treated with third generation cephalosporins (Cefoperazone, or Cefotaxime) according to protocol followed in NLI. Response to treatment was assessed considering clinical improvement, and normal AF analysis, culture, and blood culture.

\section{Statistical analysis:}

Data was statistically analyzed using SPSS (statistical package for social science) program version 13 for windows and for all the analysis a $\mathrm{p}$ value $<0.05$ was considered statistically significant:

- Data are shown as mean, range or value and 95\% confidence interval (95\% CI) and frequency and percent.

Chi square test was done for qualitative variable analysis and $\mathrm{p}$-value $<0.05$ was considered significant.

Student $\mathbf{t}$-test was done for normally distributed quantitative variables to measure mean and standard deviation and p-value $<0.05$ was considered significant.

Mann-Whitney test was done for quantitative variables which are not normally distributed and $\mathrm{p}$-value $<0.05$ was considered significant.

All data are tested with Kolmogorov-Smirnov $\mathbf{Z}$ test and most of them were found normally distributed and so presented with mean \pm SD. Parametric testes were used on doing association or correlation.

\section{Results}

60 patients with SBP were included for analysis. There were $13(21.7 \%)$ patients 
who had culture positive SBP while 47 (78.3\%) had culture negative SBP. It was observed that most of subjects were males 47 patients 35 of them were culture negative and 12 of them were culture positive. While 13 females were included 12 of them were culture negative and 1 of them was culture positive with a mean age $53.25 \pm 9.1$ for culture negative and $56.8 \pm 12.6$ for culture positive.

No significant statistical difference between culture negative and culture positive SBP as regard presentation at admission. Abdominal pain was the most frequent presentation as reported by $75 \%$ of patients, compared to $30 \%$ presented with fever, and $20 \%$ of patients presented with HE, $15 \%$ presented with hematemesis, and $1.7 \%$ by melena, and jaundice (data not tabulated)

Our study revealed that the more severe the degree of liver disease as estimated by the CTP score, the higher the positivity of the culture, however there was no significant statistical difference between culture negative and culture positive (AF culture, and $\mathrm{BC}$ ) as regard CTP (Table 4). Comparing culture negative and culture positive SBP as regards different lab parameters such as Total bilirubin, Direct bilirubin, Albumin, PT, WBCs, BUN and Creatinine, there were no significant statistical difference between them (Table 1). In the other hand a significant statistical difference reported between negative and positive blood culture in SBP as regard serum Creatinine, as shown in Table 2.

Table 1. Comparison between all studied lab parameters in culture negative and culture positive SBP.

\begin{tabular}{cccc}
\hline \multirow{2}{*}{ Studied variables } & \multicolumn{2}{c}{ SBP } & \\
\cline { 2 - 3 } & $\begin{array}{c}\text { Culture negative (no. 47) } \\
\text { Mean } \pm S D\end{array}$ & $\begin{array}{c}\text { Culture positive (no. 13) } \\
\text { Mean } \pm S D\end{array}$ & \\
\cline { 1 - 3 } Total bilirubin & $6.56 \pm 8.18$ & $6.35 \pm 6.75$ & $>0.05$ \\
Direct bilirubin & $5.05 \pm 7.26$ & $4.18 \pm 4.13$ & $>0.05$ \\
Albumin & $2.18 \pm 0.7$ & $1.88 \pm 0.39$ & $>0.05$ \\
$P T$ & $20.5 \pm 7.26$ & $22.9 \pm 8.37$ & $>0.05$ \\
WBCS & $9893.6 \pm 4995.2$ & $9046.9 \pm 4787.6$ & $>0.05$ \\
BUN & $138.4 \pm 107.5$ & $86.1 \pm 50.3$ & $>0.05$ \\
Creatinine & $2.1 \pm 1.43$ & $1.43 \pm 1.05$ & $>0.05$ \\
\hline
\end{tabular}

Table 2. Comparison between all studied lab parameters in negative blood culture and positive blood culture SBP.

\begin{tabular}{cccc}
\hline \multirow{2}{*}{ Studied variables } & \multicolumn{2}{c}{ Blood culture } & \\
\cline { 2 - 3 } & $\begin{array}{c}\text { Negative (no. 44) } \\
\text { Mean } \pm S D\end{array}$ & $\begin{array}{c}\text { Positive (no. 16) } \\
\text { Mean } \pm S D\end{array}$ & -Value \\
\hline Total bilirubin & $5.47 \pm 6.42$ & $9.38 \pm 5$ & $>0.05$ \\
Direct bilirubin & $3.91 \pm 5$ & $7.5 \pm 9.7$ & $>0.05$ \\
Albumin & $2.1 \pm 0.66$ & $2.2 \pm 0.67$ & $>0.05$ \\
$P T$ & $20.12 \pm 6.2$ & $23.6 \pm 10.12$ & $>0.05$ \\
WBCs & $9218.41 \pm 4303.1$ & $11062.5 \pm 6293.16$ & $>0.05$ \\
BUN & $111.95 \pm 90.18$ & $168.62 \pm 116.25$ & $>0.05$ \\
Creatinine & $1.73 \pm 1.23$ & $2.58 \pm 1.59$ & $<0.05^{\star}$ \\
\hline
\end{tabular}


As regards, AF chemical parameters ( $\mathrm{LDH}$, Glucose, Albumin, Total protein and SAAG) there were no significant statistical difference reported between both blood, and AF culture negative and culture positive SBP

It was noticed that the pattern of infection in ascetic fluid culture is near to that of blood culture, but Streptococci take the upper hand in blood culture and Staphylococcus species in ascetic fluid culture. Gram-positive organisms are more common in both ascetic fluid and blood culture than gram-negative organisms as shown in Figure 1.

Figure 2 show that the mortality rate was higher in culture positive SBP patients $46.2 \%$ as compared to $29.8 \%$ in culture negative group, but the difference was not significant however, the difference was significant in case of blood culture results where, $62.5 \%$ versus $22.7 \%$ died in culture positive versus culture negative patients, respectively.

Septic shock, Hepato renal syndrome (HRS), Hepatic Encephalopathy (HE)

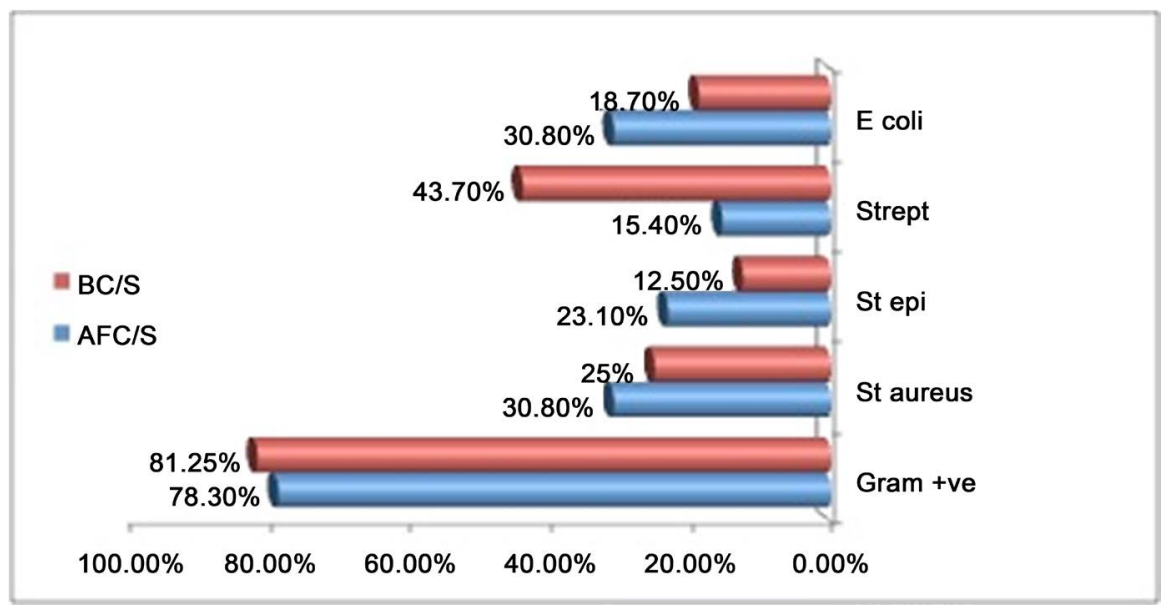

St epi: Staph epidermis; Gram +ve: Gram positive

Figure 1. Pattern of microbial infection in both ascetic fluid culture and blood culture.

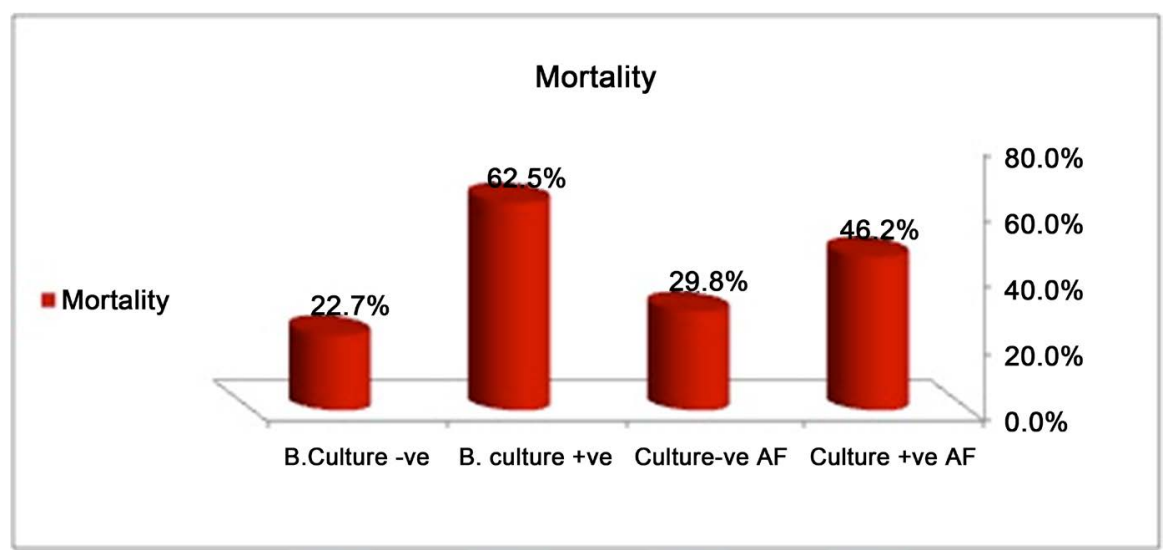

B. culture -ve: Blood culture negative; B. culture +ve: Blood culture positive; Culture -ve AF: Culture negative Ascitic Fluid; Culture +ve AF: Culture positive Ascitic Fluid

Figure 2. Mortality in both Af\&blood culture +ve and -ve patients. 


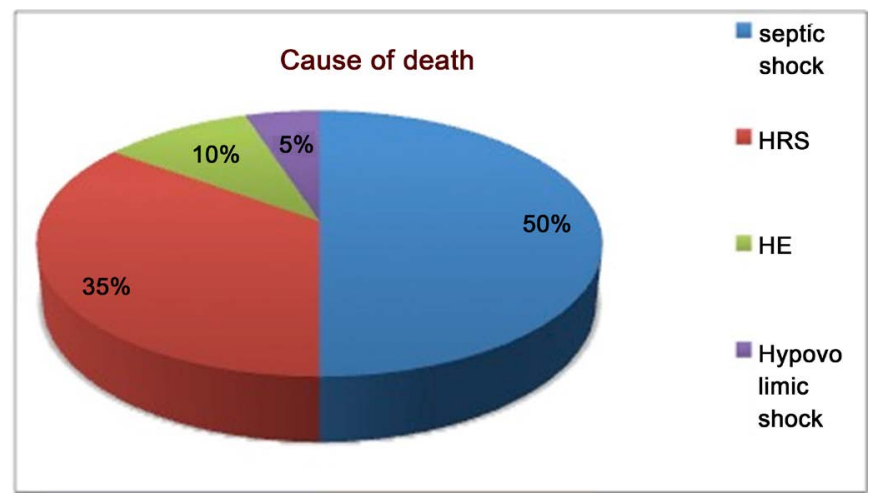

HE: Hepatic encephalopath; HRS: Hepatorenal syndrom

Figure 3. Causes of death in studied group.

and hypovolemia were the causes of death in the present study $(50 \%, 35 \%, 15 \%$, and $15 \%$ respectively) (Figure 3 ).

The total number of positive cultures was 29 cases (16 positive blood culture and 13 positive ascitic fluid culture), Staph Species represents $44.8 \%$ (13/29) with mortality rate of $61.5 \%(8 / 13)$ of all staph Infection, $27.6 \%(8 / 29)$ of all positive culture and $42 \%(8 / 20)$ of all mortalities in this study. Most of staph mortalities are resistant to cephalosporins $62.5 \%$ (5/8). Strept species represents $31 \%(9 / 29)$ with mortality rate of $44.4 \%$ (4/9) of all strept infection, $13.8 \%(4 / 29)$ of all positive culture and $20 \%(4 / 20)$ of all mortalities in this study. E.coli represents $24.2 \%(7 / 29)$ with mortality rate of $57.1 \%(4 / 7)$ of all E.coli infection, $13.8 \%$ $(4 / 29)$ of all positive culture and $20 \%$ (4/20) of all mortalities in this study (Table 3). Shifting to another antimicrobial drug (ampicillin/sulbactam, Ciprofloxacin or Levofloxacin) was noticed in about $36.7 \%$ of cases $(22 / 60)$.

\section{Discussion}

Cirrhosis is widely considered as the commonest etiology for immunodeficiency status, proceeding AIDS. Infectious complications in cirrhosis could be associated with high morbidity and mortality [12]. Spontaneous bacterial peritonitis is one of the severe and common sequels of cirrhosis with a significant mortality. Spontaneous bacterial peritonitis might be related to deterioration of reticuloendothelial system phagocytic function, leukocyte dysfunction, and depletion of serum complement level, or function [13]. The overall incidence of SBP in different studies around $8 \%-30 \%$ while the mean rate of the hospital mortality rate as high as $78 \%$ [14].

The results of the present study showed that when comparing between culture negative and culture positive SBP as regards different lab parameters such as Total bilirubin, Direct bilirubin, Albumin, PT, WBCs, BUN and Creatinine. There was no significant statistical difference between them. But there was a significant statistical difference between negative blood culture and positive blood culture in SBP as regard serum Creatinine. However, no significant statistical 
Table 3. Outcome of patients with positive cultures in relation to microorganisms.

\begin{tabular}{ccccccc}
\hline $\begin{array}{c}\text { Organism } \\
\mathbf{n}=\mathbf{2 9}\end{array}$ & $\begin{array}{c}\text { Ascitic } \\
\text { culture }\end{array}$ & $\begin{array}{c}\text { Blood } \\
\text { culture }\end{array}$ & Death & Recovery & $\begin{array}{c}\text { Resistance to } \\
\text { 3rd generation } \\
\text { cephalosporin }\end{array}$ & Sensitivity to other antibiotics \\
\hline Staph 13 cases & $724.1 \%$ & $620.7 \%$ & $827.6 \%$ & $517 \%$ & $517 \%$ & Vancomyc. Quinolone. Imepenems \\
Strept. 9 cases & $26.9 \%$ & $724.1 \%$ & $413.8 \%$ & $517 \%$ & $000 \%$ & Piperacillin tazobactam \\
E. coli 7 cases & $413.8 \%$ & $310.4 \%$ & $413.8 \%$ & $310.4 \%$ & $000 \%$ & Imepenems Augmenti. Azithromy. \\
\hline
\end{tabular}

N.B: The all percentages in this table are from all positive cultures from either ascetic fluid or blood $=29$ cases, 13 cases of which are positive ascetic culture and 16 cases are positive blood culture.

Table 4. Comparison between patient child class and score in culture negative \& culture positive SBP, and in positive \&negative blood culture (BC).

\begin{tabular}{|c|c|c|c|c|c|c|c|c|}
\hline \multirow[b]{2}{*}{ Child class } & \multicolumn{2}{|c|}{$S B P$} & \multirow[b]{2}{*}{$\begin{array}{c}\text { Total } \\
\text { n } \%\end{array}$} & \multirow[b]{2}{*}{$P$-Value } & \multicolumn{2}{|c|}{ Blood Culture } & \multirow[b]{2}{*}{$\begin{array}{c}\text { Total } \\
\text { n } \%\end{array}$} & \multirow[b]{2}{*}{$P$-Value } \\
\hline & $\begin{array}{l}\text { Culture positive } \\
\text { (no. 13) } \\
\text { n } \%\end{array}$ & $\begin{array}{c}\text { Culture negative } \\
\text { (no. 47) } \\
\text { n } \%\end{array}$ & & & $\begin{array}{c}\text { Positive } \\
\text { (no. 16) } \\
\text { n } \%\end{array}$ & $\begin{array}{c}\text { Negative } \\
\text { (no. } 44 \text { ) } \\
\text { n } \%\end{array}$ & & \\
\hline Child A & 12.1 & 00.0 & 11.7 & $>0.05$ & 12.3 & 00.0 & 11.7 & $>0.05$ \\
\hline Child B & 1225.5 & 17.7 & 1321.7 & $>0.05$ & 1022.7 & 318.75 & 1321.7 & $>0.05$ \\
\hline Child C & 3472.4 & 1292.3 & 4676.7 & $>0.05$ & 3375 & 1381.25 & 4676.7 & $>0.05$ \\
\hline Child score & $10.96 \pm 2.04$ & $11.54 \pm 1.05$ & $11.03 \pm 1.92$ & $>0.05$ & $10.93 \pm 1.87$ & $11.5 \pm 1.9$ & $11.03 \pm 1.92$ & $>0.05$ \\
\hline
\end{tabular}

difference between them as regards other lab. Parameters (Total bilirubin, Direct bilirubin, Albumin, PT, WBCs and BUN).

This was partially matching with Saleh MA et al., [15] who found that only total bilirubin was significantly higher in the positive ascitic fluid culture group. This was nearly matching with Pelletier et al., [16] who found that the only significant difference was the level of serum creatinine that was higher in the positive ascitic fluid culture group.

When comparing between culture negative and culture positive SBP as regards different AF chemical parameters (LDH, Glucose, Albumin, Total protein and SAAG) there was no significant statistical difference between them in the present study.

Although abdominal pain and fever are the cardinal features of peritonitis, SBP could be presented in cirrhotic patients with different clinical manifestation as HE, upper GI bleeding, increasing jaundice and renal function derangement or without symptoms [17].

Abdominal pain and fever represented 75\% (45/60) and 30\% (18/60) respectively in this study, Although no significant difference in clinical presentations was observed in culture positive and culture negative SBP, the incidence was higher in culture positive than culture negative SBP, $84.6 \%$ versus $72.3 \%$ for abdominal pain and $53.8 \%$ versus $23.4 \%$ for fever, reflecting a more immunocompromised state in culture positive SBP patients. 
This is similar to the conclusion reached by Pelletier G et al., [16], who found that there were no differences in clinical signs and symptoms and CTP grading between culture negative and culture positive SBP.

The incidence of culture positive SBP was $21.7 \%$ (13/60) more or less near to 23\% reported by Lubna Kamani et al., [18], other studies by Saleh MA et al., 1994 and Pelletier G et al., 1990 [15] [16], reported higher yield ranging from 50 to $71 \%$. This could be attributed to the larger amount of ascetic fluid $(10 \mathrm{cc})$ inoculated into blood culture bottles as reported by this studies. Although, the amount inoculated in this study was less than $10 \mathrm{cc}$, it could not alone explain this difference in culture yield and other unexplained factors could have participated. The incidence of culture positive SBP increases with increasing severity of liver disease reflected by CTP score; it was noticed that the more the CTP score the higher the positivity of the culture, but there was no significant statistical difference between culture negative $(10.96 \pm 2.04)$ and culture positive SBP $(11.54 \pm 1.05)$ as regard CTP score, also no significant statistical difference between blood culture negative $(10.93 \pm 1.87)$ and blood culture positive SBP (11.5 $\pm 1.9)$ as regard CTP score. This could be attributed to increasing immunodeficiency with increasing the severity of liver disease [6].

The interesting feature noted in this study is the predominance - in culture positive SBP as well as positive blood culture in SBP of gram positive organisms $69.2 \%(9 / 13)$ and $81.25(13 / 16)$ respectively as compared to gram negative organisms $30.8 \%(4 / 13)$ and $18.75 \%(3 / 16)$ respectively. Even if Staph epidermis was excluded-considered to be contaminants-still gram-positive organisms exceed gram-negative organisms. Lubna Kamani et al., [18] reported increased incidence of gram negative SBP 72.7\% (32/44) of which $61.3 \%$ (27/44) were caused by E. coli. They also reported the same pattern of infection in blood culture, $60.7 \%$ gram-negative organisms with E. coli representing $53.5 \%$ alone. Also Saleh MA et al., [15] reported prevalence of monomicrobial gram negative organisms with $E$. coli representing $2 / 3$ of cases alone.

The results of this study have been supported by the published reports by Cholongitas E et al., [19] which shows a greater predominance of gram-positive organisms in ascetic fluid culture.

Oliviero Riggio and Stefania Angeloni [20] reported that, in clinical practice, switch from Cefotaxime to another antibiotic was necessary in more than $40 \%$ of SBP cases; this is supporting the possibility that microbial etiology of SBP, as well as sensitivity to antibiotic therapy is changing. In our institute the same situation has been encountered as shifting to another antimicrobial or given a combination as (ampicillin/sulbactam, ciprofloxacin or Levofloxacin) was noticed in about $36.7 \%$ of cases $(22 / 60)$. The clinical implication of this data is to start a combination of antimicrobial therapy covering both gram positive and gram-negative organisms till culture results become available.

Blood culture represents the gold standard for diagnosis and treatment of 
bacterial infection. The incidence of positive blood culture in this study was $26.7 \%(16 / 60)$ and all had culture negative SBP. The explanation of this result is difficult but it could be related to early performance of blood culture before the circulating organism increase in the ascitic fluid, the established theory of ascetic fluid infection is that portal hypertension increases bacterial translocation to the lymphatic system, portal vein and then to the systemic circulation causing bacteremia which in turn infects ascitic fluid [21], interestingly, all culture positive SBP has negative blood culture. Other than technical factors, this could be attributed to the episodic nature of bacteremia.

This study shows that microbial pattern in either culture positive SBP or positive blood culture is nearly the same with predominance of gram-positive pathogens. Considering, the prognostic significance of early appropriate antibiotic treatment in patients with SBP, it would be important to take into consideration the results of blood culture to guide therapy of SBP with negative ascitic fluid culture.

The total number of positive cultures is 29 cases (16 positive blood culture and 13 positive ascitic fluid culture. Staph. Species represents $44.8 \%(13 / 29)$ with mortality rate of $61.5 \%(8 / 13)$ of all staph infection and $42 \%(8 / 20)$ of all over mortalities. Most of staph Mortalities are resistant to cephalosporins $62.5 \%$ $(5 / 8)$. This highlights our concept of starting combination therapy in the treatment of SBP.

The overall mortality in this study was $33.3 \%$ (20/60). This is a high incidence of mortality as compared to Lubna Kamani et al. [18] who reported that overall 23/187 (12.2\%) patients with SBP were died. Other studies by Sette et al. 1996 [22], Hurwich et al. 1993 [23], Mattos 1994 [24], Jeffries et al. 1999 [25], Thuluvath et al. 2001 [26] and Fernandez et al. 2002 [27], reported a hospital mortality up to $30 \%$ in regardless aseptic precautions; mortality was related to complications such as sudden variceal bleeding, hepatorenal syndrome, or progressive liver failure. Also Sheer TA and Runyon BA 2005 [28], reported that mortality rate of SBP is around $30 \%$. This might be due to the changing pattern of pathogen with predominance of gram-positive organisms and the high resistance to first line antimicrobial (3rd generation cephalosporins) in the setting of advanced liver disease as illustrated by the high mortality in culture positive SBP and blood culture in CTP C patients.

The mortality was higher in culture positive SBP patients $46.2 \%(6 / 13)$ as compared to $29.8 \%$ (14/47) in culture negative group, but the difference was not significant. On the other hand, the difference was significant in case of blood culture results where, $62.5 \%(10 / 16)$ versus $22.7 \%(10 / 44)$ died in culture positive versus culture negative patients, respectively.

Renal impairment is a well known prognostic factor for poor survival in patients with culture positive SBP as reported by Lubna Kamani et al., 2008 [18] and Follo A et al., 1994 [29]. In this study, the mortality was significant in patients 
with positive blood culture than in those with negative blood culture where, serum creatinine is significantly higher in the first group. Serum creatinine is not significantly different in culture positive and culture negative SBP.

Septic shock, HRS, HE and hypovolemia were the causes of death in the present study. Septic shock represents 50\% (10/20) of total mortality, while HRS $35 \%(7 / 20)$ which may be related to uncontrolled sepsis as mentioned previously. Predominance of gram-positive pathogens (especially drug resistant staph Species) was beyond this grave outcome in patients with SBP and strongly mandates alternative policy for management of patients with this potentially fatal complication.

\section{Recommendations}

The microbial pattern of organisms responsible for SBP has changed with predominance of gram-positive organisms. This shift, in addition to resistance to $3^{\text {rd }}$ generation cephalosporins necessitates culture of ascetic fluid and blood before starting antimicrobial drugs. We recommend starting empirical treatment with a combination of antimicrobials covering both Gram negative and Gram-positive organisms.

As long as the pattern of microbial infection is similar for both blood and ascetic fluid culture, blood culture results can be relied upon to guide therapy, in cases of negative ascetic fluid culture.

\section{References}

[1] Guarner, C. and Soriano, G. (2007) Bacterial Translocation and Its Consequences in Patients with Cirrhosis. European Journal of Gastroenterology \& Hepatology, 17, 27-31. https://doi.org/10.1097/00042737-200501000-00006

[2] Koulaouzidis, A., Bhat, S. and Saeed, A.A. (2009) Spontaneous Bacterial Peritonitis. World Journal of Gastroenterology, 15, 1042-1049.

[3] Bandy, S.M. (2009) Adjunct Clinical Professor of Emergency Medicine, Pikeville College School of Osteopathic Medicine and Virginia College of Osteopathic Medicine; Consulting Staff, Department of Emergency Medicine, Johnston Memorial Hospital; Medical Director, Rejuvenage Medspa; Operational Medical Director, Virginia Operations, Wings Air Rescue Ambulance Service Contributor Information and Disclosures.

[4] Amadon, M. and Arroyo, V. (2003) Ascites and Spontaneous Bacterial Peritonitis. In: Schiff, E.R., Sorrell, M.F. and Maddrey, W.C., Eds., Schiff s Diseases of the Liver, Lippincott Williams Wilkins, Philadelphia, 559-594.

[5] Garcia-Tsao, G. (2005) Bacterial Infections in Cirrhosis: Treatment and Prophylaxis. Journal of Hepatology, 42, S85-S92. https://doi.org/10.1016/j.jhep.2004.12.006

[6] Puneeta, T. and Garcia-Tsao, G. (2008) Complications of Cirrhosis. Seminars in Liver Disease, 28, 26-42.

[7] Such, J. and Runyon, B.A. (2003) Spontaneous Bacterial Peritonitis. Clinical Infectious Diseases, 27, 669-674. https://doi.org/10.1086/514940

[8] Kang, C.I., Kim, S.H., Park, W.B., Lee, K.D., Kim, H.B., Oh, M.D., Kim, E.C., Lee, 
H.S. and Choe, K.W. (2004) Clinical Outcome of Bacteremic Spontaneous Bacterial Peritonitis Due to Extended-Spectrum Beta-Lactamase-Producing Escherichia coli and Klebsiella pneumoniae. The Korean Journal of Internal Medicine, 19, 160-164. https://doi.org/10.3904/kjim.2004.19.3.160

[9] Steven, M., Alam, T., Jerome, F.X., et al. (2005) Spontaneous Bacterial Peritonitis. Medicine Journal, 2, No. 7.

[10] Wenzl, H.H., Schimpp, G., Feierl, G. and Steinwender, G. (2001) Effect of Prenatal Cortisone on Spontaneous Bacterial Translocation from Gastrointestinal Tract in Neonatal Rat. Digestive Diseases and Sciences, 48, 1171-1176.

[11] Rimola, A., Navasa, M. and Arroyo, V. (1995) Experience with Cefotaxime in the Treatment of Spontaneous Bacterial Peritonitis in Cirrhosis. Diagnostic Microbiology and Infectious Disease, 22, 141-145. https://doi.org/10.1016/0732-8893(95)00089-S

[12] Brann, S. (2006) Infectious Complications of Cirrhosis. Current Gastroenterology Reports, 4, 285-292.

[13] McCormick, P.A., Greenslade, L., Kibbler, C.C., Chin, J.K., Burroughs, A.K. and McIntyre, N. (1997) A Prospective Randomized Trial of Ceftazidime versus Netilmicin plus Mezlocillin in the Empirical Therapy of Presumed Sepsis in Cirrhotic Patients. Hepatology, 25, 833-836.

[14] Hoefs, J.C. and Runyon, B.A. (1985) Spontaneous Bacterial Peritonitis. Diseasea-Month, 31, 1, 3-48. https://doi.org/10.1016/0011-5029(85)90002-1

[15] Saleh, M.A., Abdulkadir, R.A. and Ibrahim, A.M. (1994) Spontaneous Bacterial Peritonitis and Culture Negative Neutrocytic Ascites in Patients with Non-Alcoholic Liver Cirrhosis. Journal of Gastroenterology and Hepatology, 9, 433-436.

[16] Pelletier, G., Salmon, D., Ink, O., et al. (1990) Culture-Negative Neutrocytic Ascites: A Less Severe Variant of Spontaneous Bacterial Peritonitis. Journal of Hepatology, 10, 327-331. https://doi.org/10.1016/0168-8278(90)90140-M

[17] Angeloni, S., et al. (2009) Efficacy of Current Guidelines for the Treatment of Spontaneous Bacterial Peritonitis in the Clinical Practice. World Journal of Gastroenterology, 14, 2757-2762.

[18] Kamani, L., Mumtaz, K., Ahmed, U.S, Ali, A.W. and Jafri, W. (2008) Outcomes in Culture Positive and Culture Negative Ascitic Fluid Infection in Patients with Viral Cirrhosis: Cohort Study. BMC Gastroenterology, 8, 59.

[19] Cholongitas, E., Papatheodoridis, G.V., Manesis, E.K., et al. (2006) Spontaneous Bacterial Peritonitis in Cirrhotic Patients: Is Prophylactic Propranolol Therapy Beneficial? Journal of Gastroenterology and Hepatology, 21, 581-587. https://doi.org/10.1111/j.1440-1746.2005.03982.x

[20] Riggio, O. and Angeloni, S. (2009) Ascetic Fluid Analysis for Diagnosis and Monitoring of SBP. World Journal of Gastroenterology, 15, 3845-3850.

[21] Kraehenbuhl, J.P. and Corbett, M. (2004) Immunology. Keeping the Gut Microflora at Bay. Science, 303, 1624-1625. https://doi.org/10.1126/science.1096222

[22] Sette Jr., H., Mies, S., Barros, M.F.A., et al. (1996) [Peritonite bacteriana espontânea]. Revista Paulista de Medicina, 104, 292-297.

[23] Hurwich, D.B., Lindor, K.D., Hay, J.E., et al. (1993) Prevalence of Peritonitis and the Ascitic Fluid Protein Concentration among Chronic Liver Disease Patients. The American Journal of Gastroenterology, 88, 1254-1257. 
[24] Mattos, A. (1994) [Peritonite bacteriana espontânea e suas variantes; epidemiologia diagnóstico e história natural]. GED, 13, 97-105.

[25] Jeffries, M.A., Stern, M.A., Gunaratnam, N.T., et al. (1999) Unsuspected Infection Is Infrequent in Asymptomatic Outpatients with Refractory Ascites Undergoing Therapeutic Paracentesis. The American Journal of Gastroenterology, 94, 2972-2976. https://doi.org/10.1111/j.1572-0241.1999.01445.x

[26] Thuluvath, P.J., Morss, S. and Thompson, R. (2001) Spontaneous Bacterial Peritonitis-in-Hospital Mortality, Predictors of Survival, and Health Care Costs from 1988 to 1998. The American Journal of Gastroenterology, 96, 1232-1236.

[27] Fernandez, J., Navasa, M., Gomez, J., et al. (2002) Bacterial Infections in Cirrhosis: Epidemiological Changes with Invasive Procedures and Norfloxacin Prophylaxis. Hepatology, 35, 140-148. https://doi.org/10.1053/jhep.2002.30082

[28] Sheer, T.A. and Runyon, B.A. (2005) Spontaneous Bacterial Peritonitis. Digestive Diseases, 23, 39-46. https://doi.org/10.1159/000084724

[29] Follo, A., Llovet, J.M., Navasa, M., et al. (1994) Renal Impairment after Spontaneous Bacterial Peritonitis in Cirrhosis: Incidence, Clinical Course, Predictive Factors and Prognosis. Hepatology, 20, 1495-1501. https://doi.org/10.1002/hep.1840200619

\section{Submit or recommend next manuscript to SCIRP and we will provide best service for you:}

Accepting pre-submission inquiries through Email, Facebook, LinkedIn, Twitter, etc. A wide selection of journals (inclusive of 9 subjects, more than 200 journals)

Providing 24-hour high-quality service

User-friendly online submission system

Fair and swift peer-review system

Efficient typesetting and proofreading procedure

Display of the result of downloads and visits, as well as the number of cited articles

Maximum dissemination of your research work

Submit your manuscript at: http://papersubmission.scirp.org/

Or contact ojgas@scirp.org 